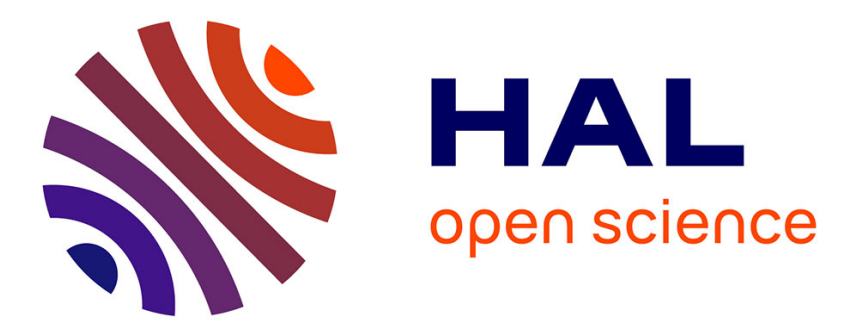

\title{
Incorporating transmission delays supported by diffusion MRI in MEG source reconstruction
}

\author{
Ivana Kojčić, Théodore Papadopoulo, Rachid Deriche, Samuel
}

Deslauriers-Gauthier

\section{- To cite this version: \\ Ivana Kojčić, Théodore Papadopoulo, Rachid Deriche, Samuel Deslauriers-Gauthier. Incorpo- rating transmission delays supported by diffusion MRI in MEG source reconstruction. ISBI 2021 - 18th IEEE International Symposium on Biomedical Imaging, Apr 2021, Nice, France. 10.1109/ISBI48211.2021.9433861 . hal-03143148}

\author{
HAL Id: hal-03143148 \\ https://hal.inria.fr/hal-03143148
}

Submitted on 16 Feb 2021

HAL is a multi-disciplinary open access archive for the deposit and dissemination of scientific research documents, whether they are published or not. The documents may come from teaching and research institutions in France or abroad, or from public or private research centers.
L'archive ouverte pluridisciplinaire HAL, est destinée au dépôt et à la diffusion de documents scientifiques de niveau recherche, publiés ou non, émanant des établissements d'enseignement et de recherche français ou étrangers, des laboratoires publics ou privés. 


\title{
INCORPORATING TRANSMISSION DELAYS SUPPORTED BY DIFFUSION MRI IN MEG SOURCE RECONSTRUCTION
}

\author{
Ivana Kojčić, Théodore Papadopoulo, Rachid Deriche and Samuel Deslauriers-Gauthier
}

\author{
Université Côte d'Azur, Inria, France
}

\begin{abstract}
White matter fibers transfer the information between brain regions with delays that are measurable with magnetoencephalography and electroencephalography (M/EEG). In the context of regularizing the dynamics of M/EEG and recovering electrical activity of the brain from M/EEG measurements, this article proposes a graph representation-based framework to solve the M/EEG inverse problem, where prior information about transmission delays supported by diffusion MRI (dMRI) are included to enforce temporal smoothness. Results of the reconstruction of brain activity from simulated MEG measurements are compared to MNE, LORETA and CGS methods and we show that our approach improves MEG source localization when compared to these three state-of-theart approaches. In addition, we show preliminary qualitative results of the proposed reconstruction method on real MEG data for a sensory-motor task.
\end{abstract}

Index Terms - MEG, source localization, inverse problems, diffusion MRI

\section{INTRODUCTION}

Integration of structural brain imaging modalities such as diffusion magnetic resonance imaging (dMRI) with functional modalities such as magnetoencephalography and electroencephalography (M/EEG) allows us to reconstruct the complex structural and functional network organization of the brain and yield a detailed picture of brain architecture and dynamics. Two complementary organizational principles of the brain represent the hallmark of its complex neural dynamics: functional segregation and functional integration [1]. Functional segregation pertains to the brain's ability for specialized information processing to occur within locally clustered and densely interconnected neural populations. Functional integration refers to the interactions among specialized brain regions distributed across different cortical areas and coordinated activation between them. Structural connectivity places constraints on which functional interactions occur in the network [1]. The union of specialized brain areas is mediated by functional integration, which happens in part through the information transfer in the long-range white matter fibers, whose physical properties introduce communication delays.
These delays are measurable with M/EEG due to its high (millisecond) temporal resolution. We can represent the brain as a graph in which the nodes are the cortical areas and edges are the physical connections between them: either local (between adjacent vertices on the cortical mesh) or non-local (long-range white matter fibers). These long-range anatomical connections can be obtained by dMRI tractography which produces a set of streamlines that represent white matter fiber bundles. Given the streamline lengths and the information conduction speed, we can estimate the propagation delays for each connection. This way dMRI can give us an insight into interaction delays of the macroscopic brain network.

Localizing and recovering electrical activity of the brain from $\mathrm{M} / \mathrm{EEG}$ measurements is known as the M/EEG inverse problem. Since there are more unknowns (brain sources) than the number of sensors, the solution is non-unique and the problem ill-posed. To obtain a unique solution, prior constraints on the characteristics of source distributions need to be introduced. Traditional linear inverse methods deploy different constraints which can favour solutions with minimum norm [2], impose smoothness constraints in space along the cortical surface [3], or time [4]. Nevertheless, the longrange anatomical connectivity is rarely taken into account. While the structural connectivity was exploited in several approaches [5, 6], temporal dynamics of the data was not considered. Very few methods include connections supported by dMRI as a prior structural information such as $[7,8]$ where source intensities are modeled with multivariate autoregressive models whose elements are constrained by dMRI-derived anatomical connections, or $[9,10]$ that are based on a probabilistic technique called maximum entropy on the mean (MEM), which explicitly use delays inferred from dMRI. However, MEM approaches suffer from high computational complexity and can be highly sensitive to the initialization of the reference distribution representing the prior knowledge of the current distribution [11].

Recently, we proposed to encapsulate delays provided by dMRI in a graph representation-based framework in a method called Connectivity-Informed M/EEG Inverse Problem (CIMIP) [12] by imposing temporal smoothness in structurally connected sources with the corresponding delays. Their potential in improving the MEG source reconstruction was shown when compared to a state-of the-art approach [5]. 
In order to further validate the robustness of the proposed method, in this work we compare our source reconstruction method to additional approaches $[2,3]$, evaluate it with additional metrics and show qualitative results on real MEG data for a sensory-motor task.

\section{FORWARD AND INVERSE M/EEG PROBLEM}

Distributed source models place the current dipoles (candidate sources) at a large number of vertices distributed on the cortical mesh, oriented normally to the cortical mantle. The relationship between source amplitudes and M/EEG measurements is expressed by the linear model

$$
\boldsymbol{M}=\boldsymbol{G} \boldsymbol{J}+\boldsymbol{E}
$$

where $M \in \mathbb{R}^{N \times T}$ is the matrix of measurements with $N$ sensors and $T$ time samples. $\boldsymbol{J} \in \mathbb{R}^{S \times T}$ is the unknown matrix of $S$ source amplitudes. Typically $N_{E E G} \approx 60-256$, $N_{M E G} \approx 150-300$ and $S \approx 10^{4}$. The gain (lead field) matrix $G \in \mathbb{R}^{N \times S}$ provides a linear relationship between source amplitudes and sensor data (i.e. the M/EEG forward solution [13]). $\boldsymbol{E} \in \mathbb{R}^{N \times T}$ is additive noise in sensor space. To recover the source amplitudes, we need to solve the following problem

$$
\min _{\boldsymbol{J}} U(\boldsymbol{J})=\min _{\boldsymbol{J}}\{F(\boldsymbol{M}, \boldsymbol{J})+\lambda P(\boldsymbol{J})\}
$$

with $F$ and $P$ being respectively fidelity and prior terms. A wide range of linear inverse methods exist in the form

$$
U(\boldsymbol{J})=\|\boldsymbol{M}-\boldsymbol{G} \boldsymbol{J}\|_{2}^{2}+\lambda\|\boldsymbol{W} \boldsymbol{J}\|_{2}^{2}
$$

Both data fidelity and prior terms are quadratic in $\boldsymbol{J}$, which yields a closed-form solution

$$
\hat{\boldsymbol{J}}=\left(\boldsymbol{G}^{\boldsymbol{T}} \boldsymbol{G}+\lambda \boldsymbol{W}^{\boldsymbol{T}} \boldsymbol{W}\right)^{-1} \boldsymbol{G}^{\boldsymbol{T}} \boldsymbol{M}
$$

These methods differ by the choice of the matrix $\boldsymbol{W} \in \mathbb{R}^{S \times S}$ which incorporates a certain regularization property. Several different priors have been proposed: (1) $\boldsymbol{W}$ as the identity matrix that penalizes the Euclidean norm of the current sources [2] (i.e. minimum norm estimates - MNE); (2) $\boldsymbol{W}$ as a depth weighting matrix, reducing the bias of MNE towards superficial sources [14]; (3) $\boldsymbol{W}$ as a discrete spatial Laplacian operator which favours smoothness between neighboring sources, also known as Low Resolution brain Electromagnetic Tomography (LORETA) [3]. Nevertheless, all $l_{2}-$ based inverse solvers suffer from smearing of even focal activation and often fail to exploit specific knowledge about the brain, such as the temporal dynamics of the underlying sources.

\subsection{Laplacian as a smoothness constraint}

We can consider brain as an undirected graph in which nodes are the current sources and edges encode physical connections between them: either local (between adjacent vertices on the cortical mesh) or non-local (long-range white matter fibers). In the LORETA approach [3] similar activity is favoured between adjacent vertices on the cortical mesh by choosing $\boldsymbol{W}$ to be the Laplacian matrix on the cortical surface, giving solution with the maximum spatial smoothness. Since this technique does not account for long-range connectivity, it was extended in the Cortical Graph Smoothing (CGS) method [5] by forming a hybrid local/nonlocal connectivity graph with $\boldsymbol{A}_{\boldsymbol{l o c}}$ (spatial adjacency on the cortical surface) and $\boldsymbol{A}_{\boldsymbol{t r}}$ (tractography-based connectivity matrix). They are used to form local $\boldsymbol{L}_{l o c}$ and tractographybased $\boldsymbol{L}_{\boldsymbol{t r}}$ graph Laplacians, resulting in the penalty term $P_{C G S}(\boldsymbol{J})=\lambda_{\text {loc }} \boldsymbol{J}^{\boldsymbol{T}} \boldsymbol{L}_{\text {loc }} \boldsymbol{J}+\lambda_{t r} \boldsymbol{J}^{\boldsymbol{T}} \boldsymbol{L}_{\boldsymbol{t r}} \boldsymbol{J}$. This way CGS penalizes the weighted sum of squared differences in activity between anatomically connected cortical patches and promotes solutions with consistent activations across them.

\section{CONNECTIVITY-INFORMED M/EEG INVERSE PROBLEM (CIMIP)}

While previous approaches exploited information about structural connectivity between different cortical areas, transmission delays were not taken into account. In order to enforce temporal smoothness between time courses of connected sources, we incorporated a modified version of the Laplacian operator as a penalty term in the minimization. We consider all time samples in a single very large problem as $\boldsymbol{m}=\tilde{\boldsymbol{G}} \boldsymbol{j}+\boldsymbol{\epsilon}$ where $\boldsymbol{m} \in \mathbb{R}^{N T}$ and $\boldsymbol{j} \in \mathbb{R}^{S T}$ are vectors of concatenated measurements and source intensities, respectively. Forward operator is now block diagonal, i.e. $\tilde{\boldsymbol{G}}=\operatorname{diag}(\boldsymbol{G}, \ldots \boldsymbol{G}) \in \mathbb{R}^{N T \times S T}$ and $\boldsymbol{\epsilon} \in \mathbb{R}^{N T}$ is additive noise. To recover electrical activity of the brain from $\mathrm{M} / \mathrm{EEG}$ measurements, we minimize the following objective function

$$
U_{\mathrm{CIMIP}}(\boldsymbol{j})=\|\boldsymbol{m}-\tilde{\boldsymbol{G}} \boldsymbol{j}\|_{2}^{2}+\lambda\left(\boldsymbol{j}^{\boldsymbol{T}} \tilde{\boldsymbol{L}} \boldsymbol{j}\right)^{2}
$$

Firstly, a binary time-dependent connectivity graph is built as $\tilde{\boldsymbol{A}}=\tilde{\boldsymbol{A}}_{\boldsymbol{l o c}}+\tilde{\boldsymbol{A}}_{\boldsymbol{t r}}$. Short-range connections are encapsulated in $\tilde{\boldsymbol{A}}_{\text {loc }}=\operatorname{diag}\left(\boldsymbol{A}_{\text {loc }}, \ldots \boldsymbol{A}_{\text {loc }}\right) \in \mathbb{R}^{S T \times S T}$. In order to include connections for different delays, we build a tractography-based graph $\tilde{\boldsymbol{A}}_{\boldsymbol{t r}} \in \mathbb{R}^{S T \times S T}$ where nonzero elements designate the presence of a long-range connection for a specific delay, which was not considered in the CGS. Using $\tilde{\boldsymbol{A}}$, the regularization matrix in (5) is formed as $\tilde{\boldsymbol{L}}=\boldsymbol{I}-\tilde{\boldsymbol{A}}$. This is a symmetric block matrix where each block corresponds to a specific delay. For every streamline $f$ connecting a pair of cortical sources, the transmission delay between them is calculated as $\Delta_{f}=\frac{l_{f}}{v} F_{s}$ where $l_{f}$ is the streamline length, $v$ is the information conduction speed and $F_{s}$ is the sampling frequency. In accordance to recent findings [15] showing that proportionality between fiber diameter and conduction velocity is $6.67 \mathrm{~ms}^{-} 1 / \mu \mathrm{m}$ and previous works of [9], information conduction speed is assumed to be constant across brain and equal to $6 \mathrm{~m} / \mathrm{s}$. 


\section{SIMULATED AND REAL DATA}

Since ground truth of source-level brain activity is unknown, it needs to be simulated in order to validate the accuracy of the proposed method. Distributed source models require representation of the cortex as a cortical mesh. Cortical surfaces were extracted from the subject's anatomical T1 MR images from the Human Connectome Project [16] dataset using Freesurfer [17]. Once the surfaces were extracted, full source space was set up using MNE-python software [18] with 4098 sources per hemisphere. The source space was parcellated into 68 neuroanatomical regions of interest according to the Desikan-Killiany atlas [19]. Cortical activity accounting for connections in both space and time is modeled using a multivariate autoregressive (MAR) models as proposed in $[7,8]$ to constrain the sources' dynamics for inverse problems. Thus, we model source-level brain activity with the following MAR model as a function of both local and long-range connections:

$$
\boldsymbol{j}_{t}=\sum_{i=1}^{p} \boldsymbol{C}_{i} \boldsymbol{j}_{t-i}+\boldsymbol{\nu}_{t}
$$

where $\boldsymbol{j}_{t}$ is the amplitude of all sources at time $t, p$ is the order of the model chosen according to delays found in the streamlines, $\boldsymbol{C}_{i}$ are the coefficient matrices, $\boldsymbol{\nu}_{t}$ is the input term chosen to resemble somatosensory evoked response potentials (ERP). MAR coefficient matrices $C_{i}$ represent the source interactions across brain areas and each of them defines the contribution of all sources at time $t-i$ to all sources at time $t$. Positions of nonzero coefficients are set according to both short-range and long-range connectivity. Assuming that only a few regions are typically active during a cognitive task, the activity is simulated for 10 randomly chosen subnetworks as a graph path with different lengths. In each of the 10 subnetworks, the number of active regions (visited graph vertices) varied from 2 to 5 . Two types of simulations were performed: (1) multiple focal sources (MFS) and (2) multiple spread sources (MSS) giving 20 simulated datasets. In MFS, only the sources in the active region having a long-range connection in the next active region were assigned the activity. In
MSS, the activity of each active source is also spread to its adjacent neighbours instantaneously. For each active source, a waveform resembling an ERP is propagated to one of the sources it is connected to via streamlines in the following region, with delays inferred from dMRI. Let us make an example of a multiple spread source simulation for 3 active regions in the left hemisphere, with the following order of activity propagation: lateral occipital sulcus $\rightarrow$ inferior parietal lobule $\rightarrow$ middle temporal gyrus. Such source-level activity is illustrated in Fig. 1(a), where we can see that different intersource delays can appear between a pair of regions. The resulting simulated MEG data using (1) is depicted in Fig. 1(b).

As a proof of concept, we tested our source reconstruction method on the real MEG data for a sensory-motor task, in which the subjects were asked to tap their right fingers after having been presented with a visual stimulus. The MEG data was provided by the HCP [20] and it was accessed using MNE-HCP toolkit (https://github.com/mne-tools/mne-hcp).

\section{RESULTS}

Results of the reconstruction of brain activity from simulated MEG measurements were compared to the MNE [2], LORETA [3] and CGS [5] methods for both simulation types. Source estimation performance is evaluated with two metrics: 1. Peak-to-Peak (PtP) localization error: the Euclidean distance between the dipole with peak magnitude $p^{*}$ in simulated (ground truth - GT) and reconstructed (REC) sources i.e. $d\left(p^{*}\right)=\left|p_{R E C}^{*}-p_{G T}^{*}\right|$ (see Fig. 2).

2. Center-of-Gravity ( $\mathrm{CoG}$ ) localization error: the Euclidean distance between the dipole that is the center of gravity (mass activity) $c^{*}$ in simulated and reconstructed sources i.e. $d\left(c^{*}\right)=\left|c_{R E C}^{*}-c_{G T}^{*}\right|$. In this case, the location of each source $\vec{r}_{i}$ is weighted by the its amplitude $\left|J_{i}\right|$. The center of mass is defined as $\vec{r}_{c^{*}}=\sum_{i}^{n}\left|J_{i}\right| \vec{r}_{i} / \sum_{i}^{n}\left|J_{i}\right|$.

Spurious dipole estimates with amplitudes $\left|J_{i}\right|$ smaller $10 \%$ of the maximum amplitude were discarded. Errors reported in Table 1 indicate the mean errors \pm standard deviation (SD), averaged over 10 subnetworks, for each simulation type.

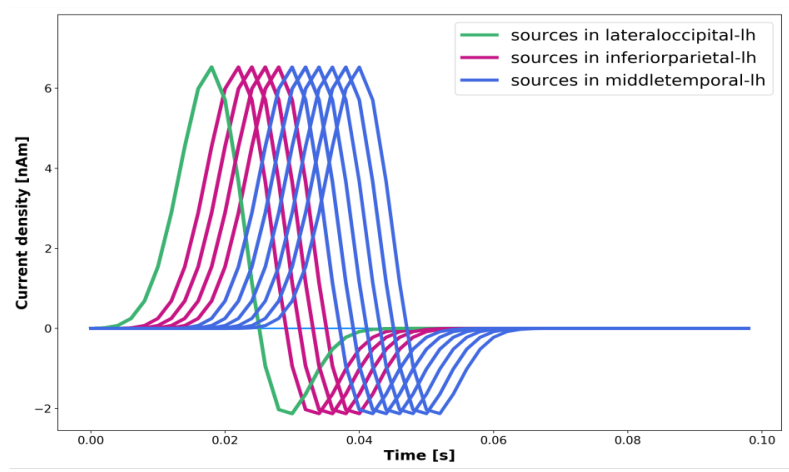

(a) Simulated source time courses

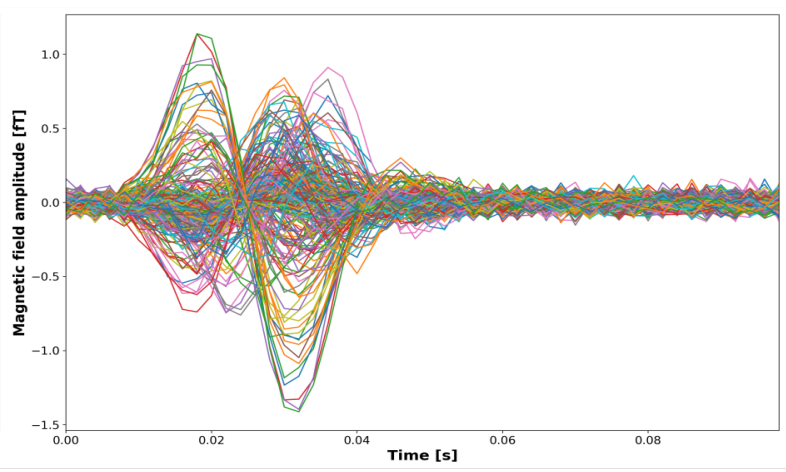

(b) The corresponding raw MEG data (with $\mathrm{SNR}=10$ )

Fig. 1. Simulated data 


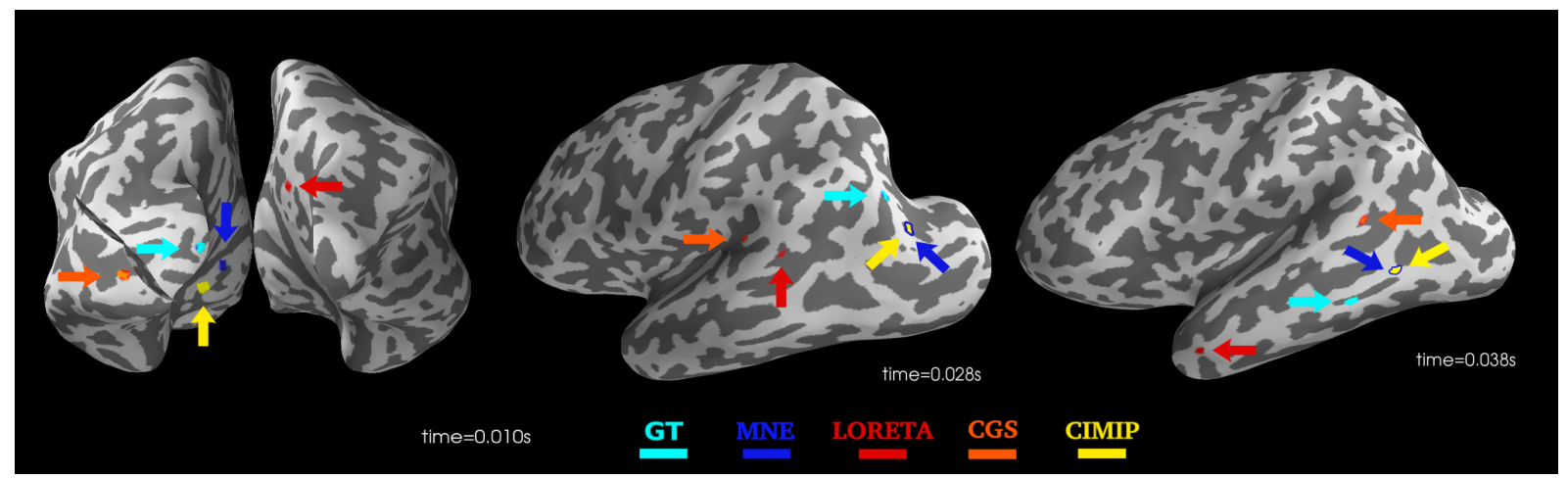

Fig. 2. Peaks in the ground truth and the reconstructions for 3 time instants: $t=0.01, t=0.028, t=0.038 \mathrm{~s}$

\begin{tabular}{|l|l|l|l|}
\hline \multicolumn{4}{|c|}{ Localization errors } \\
\hline Method & Metric & MFS & MSS \\
\hline \multirow{2}{*}{ MNE } & PtP & $31.19 \pm 13.09$ & $31.57 \pm 10.42$ \\
& CoG & $41.59 \pm 13.39$ & $40.65 \pm 13.21$ \\
\hline \multirow{2}{*}{ LORETA } & PtP & $44.79 \pm 6.29$ & $41.18 \pm 11.62$ \\
& CoG & $49.06 \pm 9.21$ & $49.77 \pm 11.63$ \\
\hline \multirow{2}{*}{ CGS } & PtP & $47.06 \pm 20.11$ & $53.59 \pm 16.96$ \\
& CoG & $54.25 \pm 6.78$ & $49.39 \pm 12.79$ \\
\hline \multirow{2}{*}{ CIMIP } & PtP & $\mathbf{2 5 . 8 3} \pm \mathbf{7 . 8 3}$ & $\mathbf{2 6 . 1 8} \pm \mathbf{5 . 6 9}$ \\
& CoG & $\mathbf{4 1 . 1 8} \pm \mathbf{1 2 . 7 7}$ & $\mathbf{3 5 . 6} \pm \mathbf{1 1 . 7 8}$ \\
\hline
\end{tabular}

Table 1. Localization errors

Regarding the real MEG data, CIMIP method recovers activations in areas associated to the experimental task, i.e. the visual, primary motor, and premotor areas, $160 \mathrm{~ms}$ after the visual stimulus (Fig. 3). Stronger activations were recovered in the occipital and parietal lobes. Weaker ones are located in precentral and postcentral regions, but also in temporal areas.

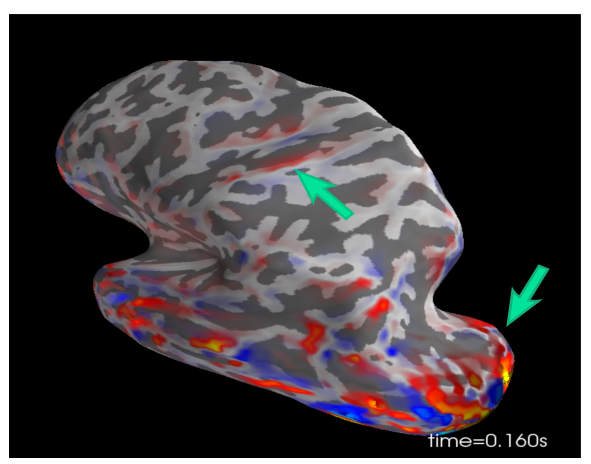

Fig. 3. CIMIP reconstruction from real MEG data

\section{CONCLUSION AND FUTURE WORK}

A graph representation-based framework was proposed to solve the M/EEG inverse problem, where prior information about conduction delays supported by dMRI are included to enforce temporal smoothness. Results on simulated synthetic dataset show that our approach improves MEG source localization when compared to three state-of-the-art approaches. Preliminary qualitative results on the real MEG data for a right hand sensory-motor task show promising results, as activations are found in the expected occipital and motor regions. Future work will include a more extensive performance evaluation on real MEG data with comparison to other methods. Incorporation of additional constraints into CIMIP (e.g. sparsity) and how they comply with different assumptions about the underlying activity will also be investigated

\section{COMPLIANCE WITH ETHICAL STANDARDS}

This research study was conducted retrospectively using human subject data made available in open access by the Human Connectome Project, WU-Minn Consortium (Principal Investigators: David Van Essen and Kamil Ugurbil; 1U54MH091657) funded by the 16 NIH Institutes and Centers that support the NIH Blueprint for Neuroscience Research; and by the McDonnell Center for Systems Neuroscience at Washington University. Ethical approval was not required as confirmed by the license attached with the open access data.

\section{ACKNOWLEDGMENTS}

This work has received funding from the European Research Council (ERC) under the European Union's Horizon 2020 research and innovation program (ERC Advanced Grant agreement No 694665: CoBCoM - Computational Brain Connectivity Mapping). The authors declare that there is no conflict of interest.

\section{REFERENCES}

[1] Giulio Tononi, Olaf Sporns, and Gerald M Edelman, "A measure for brain complexity: relating functional segregation and integration in the nervous system," Proceedings of the National Academy of Sciences, vol. 91, no. 11, pp. 5033-5037, 1994. 
[2] Matti S Hämäläinen and Risto J Ilmoniemi, “Interpreting magnetic fields of the brain: minimum norm estimates," Medical \& biological engineering \& computing, vol. 32, no. 1, pp. 35-42, 1994.

[3] Roberto D Pascual-Marqui, Christoph M Michel, and Dietrich Lehmann, "Low resolution electromagnetic tomography: a new method for localizing electrical activity in the brain," International Journal of psychophysiology, vol. 18, no. 1, pp. 49-65, 1994.

[4] Sylvain Baillet and Line Garnero, "A bayesian approach to introducing anatomo-functional priors in the eeg/meg inverse problem," IEEE transactions on Biomedical Engineering, vol. 44, no. 5, pp. 374-385, 1997.

[5] David K Hammond, Benoit Scherrer, and Simon K Warfield, "Cortical graph smoothing: a novel method for exploiting dwi-derived anatomical brain connectivity to improve eeg source estimation," IEEE transactions on medical imaging, vol. 32, no. 10, pp. 19521963, 2013.

[6] Christophe Phillips, Michael D Rugg, and Karl J Friston, "Anatomically informed basis functions for eeg source localization: combining functional and anatomical constraints," NeuroImage, vol. 16, no. 3, pp. 678695, 2002.

[7] Makoto Fukushima, Okito Yamashita, Thomas Knösche, and Masa-aki Sato, "Meg source reconstruction based on identification of directed source interactions on whole-brain anatomical networks," Neuroimage, vol. 105, pp. 408-427, 2015.

[8] Brahim Belaoucha and Theodore Papadopoulo, "Structural connectivity to reconstruct brain activation and effective connectivity between brain regions," Journal of Neural Engineering, 2020.

[9] Samuel Deslauriers-Gauthier, Jean-Marc Lina, Russell Butler, Kevin Whittingstall, Guillaume Gilbert, PierreMichel Bernier, Rachid Deriche, and Maxime Descoteaux, "White matter information flow mapping from diffusion mri and eeg," NeuroImage, vol. 201, pp. 116017, 2019.

[10] B. Belaoucha, J. Lina, M. Clerc, and T. Papadopoulo, "Mem-diffusion mri framework to solve meeg inverse problem," in 2015 23rd European Signal Processing Conference (EUSIPCO), 2015, pp. 1875-1879.

[11] Christophe Grova, Jean Daunizeau, J-M Lina, Christian G Bénar, Habib Benali, and Jean Gotman, "Evaluation of eeg localization methods using realistic simulations of interictal spikes," Neuroimage, vol. 29, no. 3, pp. 734-753, 2006.
[12] Ivana Kojčić, Théodore Papadopoulo, Rachid Deriche, and Samuel Deslauriers-Gauthier, "Connectivityinformed MEEG inverse problem," in GRAIL 2020: MICCAI Workshop on GRaphs in biomedicAl Image anaLysis, 2020.

[13] John C Mosher, Richard M Leahy, and Paul S Lewis, "Eeg and meg: forward solutions for inverse methods," IEEE Transactions on Biomedical Engineering, vol. 46, no. 3, pp. 245-259, 1999.

[14] Fa-Hsuan Lin, Thomas Witzel, Seppo P Ahlfors, Steven M Stufflebeam, John W Belliveau, and Matti S Hämäläinen, "Assessing and improving the spatial accuracy in meg source localization by depth-weighted minimum-norm estimates," Neuroimage, vol. 31, no. 1, pp. 160-171, 2006.

[15] Mark Drakesmith, Robbert Harms, Suryanarayana Umesh Rudrapatna, Greg D Parker, C John Evans, and Derek K Jones, "Estimating axon conduction velocity in vivo from microstructural mri," NeuroImage, vol. 203, pp. 116186, 2019.

[16] David C Van Essen, Stephen M Smith, Deanna M Barch, Timothy EJ Behrens, Essa Yacoub, Kamil Ugurbil, WuMinn HCP Consortium, et al., "The wu-minn human connectome project: an overview," Neuroimage, vol. 80, pp. 62-79, 2013.

[17] Bruce Fischl, "Freesurfer," Neuroimage, vol. 62, no. 2, pp. 774-781, 2012.

[18] Alexandre Gramfort, Martin Luessi, Eric Larson, Denis A Engemann, Daniel Strohmeier, Christian Brodbeck, Lauri Parkkonen, and Matti S Hämäläinen, "Mne software for processing meg and eeg data," Neuroimage, vol. 86, pp. 446-460, 2014.

[19] Rahul S Desikan, Florent Ségonne, Bruce Fischl, Brian T Quinn, Bradford C Dickerson, Deborah Blacker, Randy L Buckner, Anders M Dale, R Paul Maguire, Bradley T Hyman, et al., "An automated labeling system for subdividing the human cerebral cortex on mri scans into gyral based regions of interest," Neuroimage, vol. 31, no. 3, pp. 968-980, 2006.

[20] Linda J Larson-Prior, Robert Oostenveld, Stefania Della Penna, G Michalareas, F Prior, Abbas BabajaniFeremi, J-M Schoffelen, Laura Marzetti, Francesco de Pasquale, F Di Pompeo, et al., "Adding dynamics to the human connectome project with meg," Neuroimage, vol. 80, pp. 190-201, 2013. 